PROCEEDINGS OF THE

AMERICAN MATHEMATICAL SOCIETY

Volume 133, Number 7 , Pages 2029-2034

S 0002-9939(05)07767-1

Article electronically published on January 21, 2005

\title{
BASES OF THE CONTACT-ORDER FILTRATION OF DERIVATIONS OF COXETER ARRANGEMENTS
}

\author{
HIROAKI TERAO
}

(Communicated by John R. Stembridge)

\begin{abstract}
In a recent paper, we constructed a basis for the contact-order filtration of the module of derivations on the orbit space of a finite real reflection group acting on an $\ell$-dimensional Euclidean space. Recently M. Yoshinaga constructed another basis for the contact-order filtration. In this note we give an explicit formula relating Yoshinaga's basis to the basis we constructed earlier. The two bases turn out to be equal (up to a constant matrix).
\end{abstract}

\section{The Setup AND The MAIn RESUlt}

Let $V$ be an $\ell$-dimensional Euclidean vector space with inner product $I$. Then its dual space $V^{*}$ is equipped with the inner product $I^{*}$, which is induced by $I$. Let $S$ be the symmetric algebra of $V^{*}$ over $\mathbb{R}$. Identify $S$ with the algebra of polynomial functions on $V$. Let $\operatorname{Der}_{S}$ be the $S$-module of $\mathbb{R}$-linear derivations of $S$. When $X_{1}, \cdots, X_{\ell}$ denote a basis for $V^{*}$, the partial derivations $\partial_{i}:=\partial / \partial X_{i}$ with respect to $X_{i}(1 \leq i \leq \ell)$ naturally form a basis for $\operatorname{Der}_{S}$ over $S$. Let $K$ be the field of quotients of $S$ and $\operatorname{Der}_{K}$ be the $K$-vector space of $\mathbb{R}$-linear derivations of $K$. Then the partial derivations $\partial_{i}(1 \leq i \leq \ell)$ naturally form a basis for $\operatorname{Der}_{K}$ over $K$.

Let $W$ be a finite irreducible orthogonal reflection group (a Coxeter group) acting on $V$. The Coxeter group $W$ naturally acts on $V^{*}, S$ and $\operatorname{Der}_{S}$. The $W$ invariant subring of $S$ is denoted by $R$. Then it is classically known [1, V.5.3, Theorem 3] that there exist algebraically independent homogeneous polynomials $P_{1}, \cdots, P_{\ell} \in R$ with $\operatorname{deg} P_{1} \leq \cdots \leq \operatorname{deg} P_{\ell}$, which are called basic invariants, such that

$$
R=\mathbb{R}\left[P_{1}, \cdots, P_{\ell}\right]
$$

The primitive derivation $D \in \operatorname{Der}_{R}$ is characterized by

$$
D P_{i}= \begin{cases}1 & \text { for } i=\ell \\ 0 & \text { otherwise }\end{cases}
$$

Received by the editors June 25, 2002 and, in revised form, March 1, 2004.

2000 Mathematics Subject Classification. Primary 32S22.

The author was partially supported by the Grant-in-aid for scientific research (Nos. 14340018 and 13874005), the Ministry of Education, Sports, Science and Technology, Japan. 
Write $\mathbf{P}=\left(P_{1}, \ldots, P_{\ell}\right)$. Let $J(\mathbf{P})$ denote the Jacobian matrix $\left[\partial P_{j} / \partial X_{i}\right]_{i j}$. Define $\Delta:=\operatorname{det} J(\mathbf{P})$. Then $\Delta$ is an anti-invariant [1, V.5.4] and $\Delta^{2} \in R$. Let

$$
I: \operatorname{Der}_{R} \times \operatorname{Der}_{R} \rightarrow \frac{1}{\Delta^{2}} R
$$

be the symmetric $R$-bilinear form induced by $I$. Let

$$
\begin{gathered}
\nabla: \operatorname{Der}_{R} \times \operatorname{Der}_{R} \longrightarrow \frac{1}{\Delta^{2}} \operatorname{Der}_{R}, \\
(X, Y) \longmapsto \nabla X Y
\end{gathered}
$$

be the Levi-Civita connection with respect to $I$. Since $\frac{\partial}{\partial P_{j}} \in \frac{1}{\Delta} \operatorname{Der}_{S}$ by Cramer's rule, one can embed $\operatorname{Der}_{R}$ into $\operatorname{Der}_{K}$. Extend the Levi-Civita connection naturally to

$$
\nabla: \operatorname{Der}_{K} \times \operatorname{Der}_{K} \longrightarrow \operatorname{Der}_{K}
$$

Note that $\nabla_{\xi}(\eta)=\sum_{i} \xi\left(\eta\left(X_{i}\right)\right) \partial_{i}$ because each $I\left(\partial_{i}, \partial_{j}\right)$ is constant.

In [6], we introduced derivations $\xi_{1}^{(m)}, \ldots, \xi_{\ell}^{(m)} \in \operatorname{Der}_{S}$ for $m \geq 0$ by

$$
\left(\xi_{1}^{(m)}, \ldots, \xi_{\ell}^{(m)}\right):=\left\{\begin{array}{l}
\left(\frac{\partial}{\partial P_{1}}, \ldots, \frac{\partial}{\partial P_{\ell}}\right) J(\mathbf{P})^{T} A J\left(D^{k}[\mathbf{X}]\right)^{-1} \text { if } m=2 k, \\
\left(\frac{\partial}{\partial P_{1}}, \ldots, \frac{\partial}{\partial P_{\ell}}\right) J(\mathbf{P})^{T} A J\left(D^{k}[\mathbf{X}]\right)^{-1} J(\mathbf{P}) \text { if } m=2 k+1 .
\end{array}\right.
$$

Here $A:=\left[I^{*}\left(X_{i}, X_{j}\right)\right]_{i j}$ and $J\left(D^{k}[\mathbf{X}]\right):=\left[\partial\left(D^{k}\left(X_{j}\right) / \partial X_{i}\right]_{i j}\left(D^{k}:=D \circ D \circ \cdots \circ\right.\right.$ $D$ ( $k$ times $)$ ). Let $\mathcal{A}$ be the Coxeter arrangement determined by the Coxeter group $W: \mathcal{A}$ is the set of reflecting hyperplanes. Choose for each hyperplane $H \in \mathcal{A}$ a linear form $\alpha_{H} \in V^{*}$ such that $H=\operatorname{ker}\left(\alpha_{H}\right)$. The derivations $\xi_{1}^{(m)}, \ldots, \xi_{\ell}^{(m)} \in \operatorname{Der}_{S}$ were constructed in [6] so that they may form a basis for the $S$-module [10]

$$
\mathrm{D}^{(m)}(\mathcal{A}):=\left\{\theta \in \operatorname{Der}_{S} \mid \theta\left(\alpha_{H}\right) \in S \alpha_{H}^{m} \text { for any } H \in \mathcal{A}\right\}
$$

for each nonnegative integer $m$. The filtration

$$
\operatorname{Der}_{S}=\mathrm{D}^{(0)}(\mathcal{A}) \supset \mathrm{D}^{(1)}(\mathcal{A}) \supset \mathrm{D}^{(2)}(\mathcal{A}) \supset \cdots
$$

of $\operatorname{Der}_{S}$ is called the contact-order filtration. Define

$$
\mathcal{H}^{(k)}=\mathrm{D}^{(2 k-1)}(\mathcal{A}) \cap \operatorname{Der}_{R}
$$

for each $k \geq 1$. Let $\mathcal{H}^{(0)}=\operatorname{Der}_{R}$. Then we have a filtration

$$
\operatorname{Der}_{R}=\mathcal{H}^{(0)} \supset \mathcal{H}^{(1)} \supset \mathcal{H}^{(2)} \supset \cdots
$$

of $\operatorname{Der}_{R}$. This filtration is known [7] to be equal to the Hodge filtration introduced by K. Saito [4]. The derivations $\xi_{1}^{(2 k-1)}, \ldots, \xi_{\ell}^{(2 k-1)} \in \operatorname{Der}_{R}$ form an $R$-basis for $\mathcal{H}^{(k)}$ for $k \geq 1$. Therefore the contact-order filtration, retricted to $\operatorname{Der}_{R}$, gives the Hodge filtration. Define

$$
T:=\{f \in R \mid D f=0\}=\mathbb{R}\left[P_{1}, \ldots, P_{\ell-1}\right] .
$$

Then the covariant derivative $\nabla_{D}: \operatorname{Der}_{K} \longrightarrow \operatorname{Der}_{K}$ is $T$-linear. The Hodge filtration was originally defined so that the $T$-linear map

$$
\nabla_{D}^{k}:=\nabla_{D} \circ \cdots \circ \nabla_{D}(k \text { times }): \mathcal{H}^{(k)} \longrightarrow \mathcal{H}^{(0)}=\operatorname{Der}_{R}
$$

is bijective [4]. Thus we may define $\nabla_{D}^{-k} \xi \in \mathcal{H}^{(k)}$ for any $\xi \in \operatorname{Der}_{R}$ and $k \geq 0$. In [8] M. Yoshinaga proved the following theorem. 
Theorem 1.1. ([8, Theorem 6]) Let $k \geq 0$ and

$$
E:=\sum_{i} X_{i} \partial_{i}=\sum_{i}\left(\operatorname{deg} P_{i}\right)^{-1} P_{i}\left(\partial / \partial P_{i}\right)
$$

be the Euler derivation. Suppose that $\xi_{1}, \ldots, \xi_{\ell}$ are a basis for $\mathrm{D}^{(1)}(\mathcal{A})$. Then

(1) the derivations $\nabla_{\xi_{1}} \nabla_{D}^{-k} E, \ldots, \nabla_{\xi_{\ell}} \nabla_{D}^{-k} E$ form a basis for $\mathrm{D}^{(2 k-1)}(\mathcal{A})$ over $S$, and

(2) the derivations $\nabla_{\partial_{1}} \nabla_{D}^{-k} E, \ldots, \nabla_{\partial_{\ell}} \nabla_{D}^{-k} E$ form a basis for $\mathrm{D}^{(2 k)}(\mathcal{A})$ over $S$.

It thus seems natural to ask how the basis above constructed by Yoshinaga is related to the basis $\xi_{1}^{(m)}, \ldots, \xi_{\ell}^{(m)}$ given in [6]. The following theorem answers this question:

Theorem 1.2. Let $k \geq 0$. Then

$$
\left(\xi_{1}^{(2 k+1)}, \ldots, \xi_{\ell}^{(2 k+1)}\right)=(-1)^{k}\left(\nabla_{\xi_{1}^{(1)}} \nabla_{D}^{-k} E, \ldots, \nabla_{\xi_{\ell}^{(1)}} \nabla_{D}^{-k} E\right)
$$

and

$$
\left(\xi_{1}^{(2 k)}, \ldots, \xi_{\ell}^{(2 k)}\right)=(-1)^{k}\left(\nabla_{\partial_{1}} \nabla_{D}^{-k} E, \ldots, \nabla_{\partial_{\ell}} \nabla_{D}^{-k} E\right) A .
$$

So the two bases turn out to be equal up to a constant matrix.

The significance of Theorem 1.2 is as follows: Recently M. Yoshinaga 9] affirmatively settled the Edelman-Reiner conjecture, which asserts that the cones of the extended Shi/Catalan arrangements are free. In other words, there exist basic derivations for each extended Shi/Catalan arrangement. However, a formula for basic derivations is still unknown. Since the derivations $\xi_{i}^{(m)}(i=1, \ldots, \ell)$ are the principal (= highest degree) parts of basic derivations, Theorem 1.2 can be interpreted as a differential-geometric formula for the "principal parts." So it may suggest the existence of a differential-geometric formula for the whole basic derivations, including the "non-principal part." One may also regard Theorem 1.2 as a very explicit algebraic description of the derivations in the right-hand side. They are, as mentioned above, when $m$ is odd, bases for the Hodge filtration, which is the key to defining the flat structure on the orbit space $V / W$ in [5]. The flat structure is called the Frobenius manifold structure from the viewpoint of topological field theory [2].

\section{Proof}

We will prove Theorem 1.2 in this section. First we show the following.

Lemma 2.1. For $k \geq 1$ and $\xi \in \operatorname{Der}_{K}$, we have

$$
\nabla_{D}^{k} \circ \nabla_{\xi}-\nabla_{\xi} \circ \nabla_{D}^{k}=k \nabla_{D}^{k-1} \circ \nabla_{[D, \xi]} .
$$

Proof. We use an induction on $k$. When $k=1$, the lemma asserts that

$$
\nabla_{D} \circ \nabla_{\xi}-\nabla_{\xi} \circ \nabla_{D}=\nabla_{[D, \xi]}
$$

which is the integrable property of the Levi-Civita connection $\nabla$. Let $k>1$. We have

$$
\begin{aligned}
\nabla_{D}^{k} \circ \nabla_{\xi} & =\nabla_{D}^{k-1} \circ\left(\nabla_{\xi} \circ \nabla_{D}+\nabla_{[D, \xi]}\right) \\
& =\left(\nabla_{D}^{k-1} \circ \nabla_{\xi} \circ \nabla_{D}+\nabla_{D}^{k-1} \circ \nabla_{[D, \xi]}\right. \\
& =\left(\nabla_{\xi} \circ \nabla_{D}^{k-1}+(k-1) \nabla_{D}^{k-2} \circ \nabla_{[D, \xi]} \circ \nabla_{D}+\nabla_{D}^{k-1} \circ \nabla_{[D, \xi]}\right. \\
& =\nabla_{\xi} \circ \nabla_{D}^{k}+(k-1) \nabla_{D}^{k-2} \circ \nabla_{[D, \xi]} \circ \nabla_{D}+\nabla_{D}^{k-1} \circ \nabla_{[D, \xi]}
\end{aligned}
$$


by using the induction assumption. Since $[D,[D, \xi]]=0$, we obtain

$$
\nabla_{D} \circ \nabla_{[D, \xi]}=\nabla_{[D, \xi]} \circ \nabla_{D}
$$

This implies that

$$
\nabla_{D}^{k} \circ \nabla_{\xi}=\nabla_{\xi} \circ \nabla_{D}^{k}+k \nabla_{D}^{k-1} \circ \nabla_{[D, \xi]} .
$$

Recall the $W$-invariant inner product $I^{*}: V^{*} \times V^{*} \longrightarrow \mathbb{R}$. Let $\Omega_{R}^{1}$ denote the $R$-module of Kähler differentials. Let

$$
I^{*}: \Omega_{R}^{1} \times \Omega_{R}^{1} \longrightarrow R
$$

be the symmetric $R$-bilinear form induced by $I^{*}$. Let

$$
G:=\left[I^{*}\left(d P_{i}, d P_{j}\right)\right]_{i j}=J(\mathbf{P})^{T} A J(\mathbf{P}) .
$$

Define

$$
B^{(k)}:=-J(\mathbf{P})^{T} A J\left(D^{k}[\mathbf{X}]\right) J\left(D^{k-1}[\mathbf{X}]\right)^{-1} J(\mathbf{P})
$$

for $k \geq 1$ as in [6]. Then we have

Lemma 2.2. (1) Every entry of $B^{(k)}$ lies in $T ; D\left[B^{(k)}\right]=0$,

(2) $\operatorname{det} B^{(k)} \in \mathbb{R}^{*}$,

(3) $D[G]=B^{(1)}+\left(B^{(1)}\right)^{T}$,

(4) $B^{(k+1)}=B^{(1)}+k D[G]$.

Proof. By [6, Lemmas 3.3-3.6, Remark 3.7].

The following proposition is one of the main results in [7]:

Proposition 2.3. For $k \geq 1$,

$$
\left(\nabla_{D}^{k} \xi_{1}^{(2 k-1)}, \ldots, \nabla_{D}^{k} \xi_{\ell}^{(2 k-1)}\right)=(-1)^{k-1}\left(\frac{\partial}{\partial P_{1}}, \ldots, \frac{\partial}{\partial P_{\ell}}\right) B^{(k)}
$$

In the rest of this note, let $\left(\xi_{1}, \ldots, \xi_{\ell}\right)$ denote $\left(\xi_{1}^{(1)}, \ldots, \xi_{\ell}^{(1)}\right)$ for simplicity. Note that

$$
\left(\xi_{1}, \ldots, \xi_{\ell}\right)=\left(\frac{\partial}{\partial P_{1}}, \ldots, \frac{\partial}{\partial P_{\ell}}\right) G .
$$

\section{Lemma 2.4 .}

$$
\left(\left[D, \xi_{1}\right], \ldots\left[D, \xi_{\ell}\right]\right)=\left(\frac{\partial}{\partial P_{1}}, \ldots, \frac{\partial}{\partial P_{\ell}}\right) D[G]=\left(\nabla_{D} \xi_{1}, \ldots, \nabla_{D} \xi_{\ell}\right)\left(B^{(1)}\right)^{-1} D[G] .
$$

Proof. The first equality easily follows from

$$
\left[D, \xi_{j}\right]\left(P_{i}\right)=D \circ \xi_{j}\left(P_{i}\right)-\xi_{j} \circ D\left(P_{i}\right)=D \circ \xi_{j}\left(P_{i}\right)=D\left[I^{*}\left(d P_{i}, d P_{j}\right)\right] .
$$

For the second equality, apply Proposition 2.3 when $k=1$.

Lemma 2.5. For any $\eta \in \operatorname{Der}_{K}$,

$$
\left(\nabla_{\xi_{1}} \eta, \ldots, \nabla_{\xi_{\ell}} \eta\right)=\left(\nabla_{\partial_{1}} \eta, \ldots, \nabla_{\partial_{\ell}} \eta\right) A J(\mathbf{P}) .
$$


Proof. Since both sides are additive with respect to $\eta$, we may assume $\eta=f \partial_{j}$ for some $j$ and $f \in K$. Then we have

$$
\begin{aligned}
\left(\nabla_{\xi_{1}} \eta, \ldots, \nabla_{\xi_{\ell}} \eta\right) & =\left(\nabla_{\xi_{1}}\left(f \partial_{j}\right), \ldots, \nabla_{\xi_{\ell}}\left(f \partial_{j}\right)\right) \\
& =\left(\xi_{1}(f) \partial_{j}, \ldots, \xi_{\ell}(f) \partial_{j}\right) \\
& =\left(\left(\partial_{1} f\right) \partial_{j}, \ldots,\left(\partial_{\ell} f\right) \partial_{j}\right) A J(\mathbf{P}) \\
& =\left(\nabla_{\partial_{1}}\left(f \partial_{j}\right), \ldots, \nabla_{\partial_{\ell}}\left(f \partial_{j}\right)\right) A J(\mathbf{P}) \\
= & \left(\nabla_{\partial_{1}} \eta, \ldots, \nabla_{\partial_{\ell}} \eta\right) A J(\mathbf{P}) .
\end{aligned}
$$

Proof of Theorem 1.2. When $k=0$, the formulas are obviously true. Let $k \geq 1$. Let $\xi \in\left\{\xi_{1}, \ldots, \xi_{\ell}\right\}$. Apply the identity in Lemma 2.1 to $\nabla_{D}^{-k} E$ to get

$$
\left(\nabla_{D}^{k} \circ \nabla_{\xi}\right)\left(\nabla_{D}^{-k} E\right)-\nabla_{\xi} E=k\left(\nabla_{D}^{k-1} \circ \nabla_{[D, \xi]}\right)\left(\nabla_{D}^{-k} E\right)=k \nabla_{D}^{-1}\left(\nabla_{[D, \xi]} E\right) .
$$

Since $\nabla_{\eta} E=\eta$ for any $\eta \in \operatorname{Der}_{K}$, one obtains

$$
\nabla_{D}^{k}\left(\nabla_{\xi} \nabla_{D}^{-k} E\right)-\xi=k \nabla_{D}^{-1}[D, \xi]
$$

and thus

$$
\nabla_{D}^{k+1}\left(\nabla_{\xi} \nabla_{D}^{-k} E\right)=\nabla_{D} \xi+k[D, \xi] .
$$

On the other hand, Lemma 2.4 asserts

$$
\left(\left[D, \xi_{1}\right], \ldots\left[D, \xi_{\ell}\right]\right)=\left(\nabla_{D} \xi_{1}, \ldots, \nabla_{D} \xi_{\ell}\right)\left(B^{(1)}\right)^{-1} D[G] .
$$

So we have

$$
\begin{aligned}
& \left(\nabla_{D}^{k+1}\left(\nabla_{\xi_{1}} \nabla_{D}^{-k} E\right), \ldots, \nabla_{D}^{k+1}\left(\nabla_{\xi_{\ell}} \nabla_{D}^{-k} E\right)\right) \\
= & \left(\nabla_{D} \xi_{1}, \ldots, \nabla_{D} \xi_{\ell}\right)+k\left(\nabla_{D} \xi_{1}, \ldots, \nabla_{D} \xi_{\ell}\right)\left(B^{(1)}\right)^{-1} D[G] \\
= & \left(\nabla_{D} \xi_{1}, \ldots, \nabla_{D} \xi_{\ell}\right)\left(B^{(1)}\right)^{-1}\left(B^{(1)}+k D[G]\right) \\
= & \left(\nabla_{D} \xi_{1}, \ldots, \nabla_{D} \xi_{\ell}\right)\left(B^{(1)}\right)^{-1} B^{(k+1)} \\
= & \left(\partial / \partial P_{1}, \ldots, \partial / \partial P_{\ell}\right) B^{(k+1)} \\
= & (-1)^{k}\left(\nabla_{D}^{k+1} \xi_{1}^{(2 k+1)}, \ldots, \nabla_{D}^{k+1} \xi_{\ell}^{(2 k+1)}\right)
\end{aligned}
$$

by Lemma 2.2 and Proposition 2.3 . This proves the first formula.

For the second formula, compute

$$
\left(\nabla_{\xi_{1}} \nabla_{D}^{-k} E, \ldots, \nabla_{\xi_{\ell}} \nabla_{D}^{-k} E\right)=\left(\nabla_{\partial_{1}} \nabla_{D}^{-k} E, \ldots, \nabla_{\partial_{\ell}} \nabla_{D}^{-k} E\right) A J(\mathbf{P})
$$

by Lemma 2.5. Applying the first formula, we get

$$
\begin{aligned}
\left(\xi_{1}^{(2 k)}, \ldots, \xi_{\ell}^{(2 k)}\right) J(\mathbf{P}) & =\left(\xi_{1}^{(2 k+1)}, \ldots, \xi_{\ell}^{(2 k+1)}\right) \\
& =(-1)^{k}\left(\nabla_{\xi_{1}} \nabla_{D}^{-k} E, \ldots, \nabla_{\xi_{\ell}} \nabla_{D}^{-k} E\right) \\
& =(-1)^{k}\left(\nabla_{\partial_{1}} \nabla_{D}^{-k} E, \ldots, \nabla_{\partial_{\ell}} \nabla_{D}^{-k} E\right) A J(\mathbf{P})
\end{aligned}
$$

This completes the proof of Theorem 1.2 . 


\section{REFERENCES}

[1] Bourbaki, N.: Groupes et Algèbres de Lie. Chapitres 4,5 et 6, Hermann, Paris, 1968 MF 0240238 (39:1590)

[2] Dubrovin, B.: Geometry of 2D topological field theories. In: "Integrable systems and quantum groups" (ed. Francaviglia, M., Greco, S.), Lectures at C.I.M.E., 1993, LNM 1620, Springer, Berlin-Heidelberg-New York, 1996, pp. 120-348 MR1397274 (97d:58038)

[3] Orlik, P., Terao, H.: Arrangements of Hyperplanes. Grundlehren der Math. Wiss. 300, Springer Verlag, 1992 MR1217488 (94e:52014)

[4] Saito, K.: On a linear structure of the quotient variety by a finite reflexion group. RIMS Kyoto preprint 288, 1979 = Publ. Res. Inst. Math. Sci. 29 (1993) 535-579 MR 1245441 (94k:32059)

[5] Saito, K.: Finite reflection groups and related geometry (A motivation to the period mapping for primitive forms). preprint, 2000

[6] Terao, H.: Multiderivations of Coxeter arrangements. Inventiones Math., 148 (2002) 659674 MR $1908063(2003 \mathrm{~h}: 20074)$

[7] Terao, H.: The Hodge filtration and the contact-order filtration of derivations of Coxeter arrangements. preprint 2002 (math.CO/0205058)

[8] Yoshinaga, M.: The primitive derivation and freeness of multi-Coxeter arrangements. Proc. Japan Acad. Ser. A Math. Sci. 78 (2002), no. 7, 116-119 MR1930214 (2003k:32039)

[9] Yoshinaga, M.: Characterization of a free arrangement and conjecture of Edelman and Reiner. Inventiones Math., 157 (2004), 449-454. MR2077250

[10] Ziegler, G. M.: Multiarrangements of hyperplanes and their freeness. In: Singularities. Contemporary Math. 90, Amer. Math. Soc., 1989, pp. 345-359 MR1000610 (90e:32015)

Department of Mathematics, Tokyo Metropolitan University, Minami-Ohsawa, HaCHIOJI, TOKYO 192-0397, JAPAN 